\title{
NEUTRINO ASTRONOMY AT THE SOUTH POLE
}

\author{
D.J. BOERSMA * \\ The IceCube Project, \\ 222 West Washington Avenue, \\ Madison, Wisconsin, USA \\ E-mail: boersma@icecube.wisc.edu
}

\begin{abstract}
A brief overview of AMANDA and IceCube is presented, with an introduction into
\end{abstract} the basic physics and detection principles and a small selection of the results.

\section{Introduction}

More than a hundred years after cosmic rays were discovered by Victor Hess their origin is still a mystery. Their energy spectrum extends to $10^{11} \mathrm{GeV}$, many orders of magnitude higher than can be reached with the current particle accelerators. There are various models and ideas about how cosmic rays acquire such high energies, such as Fermi acceleration in the jets of so-called Active Galactic Nuclei or in the shells of matter blasted away during a gamma-ray burst (GRB), in a Supernova remnant or in some other explosive astrophysical event. There are also models involving topological defects $^{1}$ such as magnetic monopoles created during the Big Bang. All these hypotheses may turn out to be wrong or insufficient. Whatever the outcome, the cosmic ray puzzle will teach us something fundamentally new about the Universe.

In the quest to solve this puzzle it is important to collect as many independent observations as possible. Measurements of, or constraints on, the flux of high energy neutrinos complement the existing and ongoing measurements of hadronic and electromagnetic cosmic rays. This is the main purpose of the AMANDA and IceCube experiments.

In many of the models for cosmic ray acceleration, hadrons (typically protons) are accelerated, which may collide with stellar or interstellar ma-

*On behalf of the icecube collaboration. Full author list:
http://icecube.wisc.edu/science/publications/llwi2006.html


terial, thereby producing pions. The neutral pions decay into photons (high energy gamma rays), while the decay of each charged pion produces in total three neutrinos $\left(\nu_{\mu}, \bar{\nu}_{\mu}\right.$ and $\left.\nu_{\mathrm{e}} / \bar{\nu}_{\mathrm{e}}\right)$, which may carry a significant fraction of the energy of the initial accelerated particle. This scenario is called the beam dump model.

There is a theoretical upper bound on the energy of cosmic rays - the so-called GZK-cutoff ${ }^{2}$ - due to the presence of $3 \mathrm{~K}$ cosmic microwave background (CMB): e.g. a proton with more than $50 \mathrm{EeV}$ can be excited to a $\Delta$ resonance by a CMB photon. The decay of a (charged) $\Delta$ resonance will result in (extremely high energy) neutrinos.

A particularly interesting aspect of neutrinos is that they are unlikely to get deflected or absorbed on their long journey from their generation to our detector, so that with their direction they point back to their origin and we can indeed speak about "neutrino astronomy".

\section{Detection of high energy neutrinos in ice}

When a high energy muon neutrino undergoes a charged current interaction in ice it converts into a muon. This muon keeps most of the neutrino's energy and radiates Cherenkov light. By populating the ice with photomultiplier tubes (PMTs) and measuring arrival times of the Cherenkov photons, the direction of the long muon tracks $(\sim 1 \mathrm{~km}$ at $E=200 \mathrm{GeV})$ can be reconstructed to an accuracy of $2^{\circ}-3^{\circ}$ in AMANDA (expected better than $1^{\circ}$ in IceCube). For $E_{\nu}=1 \mathrm{TeV}$ the angle between the muon and the original neutrino is of the order of $1^{\circ}$. There is a high rate of down-going muons coming from cosmic ray showers in the atmosphere directly above the South Pole; this rate is $10^{6}$ times higher than the expected neutrino rate, so except for very high energies neutrinos can only be identified if they were coming through the Earth (up-going).

A charged current interaction by an electron or tau neutrino leads to an electromagnetic cascade. A neutral current interaction by any neutrino leads to a hadronic cascade. Cascades are relatively short (typically several tens of meters), so the angular resolution is much worse than for muons $\left(30^{\circ}-40^{\circ}\right.$ in AMANDA), but the energy resolution is better.

An important role in the reconstruction is played by optical properties of the ice ${ }^{4}$. These vary with the concentration of insoluble dust, which tracks climatological variations in the last 70,000 years. For the wavelengths around $400 \mathrm{~nm}$ the absorption length $\lambda_{a}$ ranges from 50 to 130 meter (average $110 \mathrm{~m}$ ) and the effective scattering length $\lambda_{\text {eff }}$ ranges from 6 to $30 \mathrm{~m}$ 
(average $20 \mathrm{~m}$ ).

\section{Detector hardware}

\section{$A M A N D A$}

The Antarctic Muon And Neutrino Detector Array (AMANDA) consists of 19 strings with 20-42 optical modules (OMs) per string, frozen in ice at depths of $1500-2000 \mathrm{~m}$. The strings are distributed over a roughly circular area with a diameter of $\sim 200 \mathrm{~m}$. Each of the $677 \mathrm{OMs}$ consists of an 8 inch photomultiplier tube enclosed in a pressure resistant glass sphere and is connected to the surface electronics by a twisted pair, coaxial and/or optical cable.

\section{IceCube}

IceCube, when completed, will have 70 strings, each with 60 Digital Optical Modules (DOMs) spaced by $17 \mathrm{~m}$. The strings are arranged in a hexagonal pattern spanning an area of about $1 \mathrm{~km}^{2}$. The distance between neighboring strings is $120 \mathrm{~m}$. AMANDA, which has inter-string distances of $30-50 \mathrm{~m}$, will be enclosed by IceCube and will be kept in operation, serving as a low energy sub-array. The signals of the PMTs are read out and digitized inside each DOM itself, thus avoiding signal distortion in the long cables to the surface. A full description of the design can be found in Ref. 5 .

During the austral summer of 2004-2005 the first IceCube string was deployed and commissioned, demonstrating that the hot-water drill, the deployment hardware and all detector components perform according to specifications ${ }^{6}$. During the 2005-2006 season 8 more strings were deployed, so currently IceCube has 9 strings with in total 540 DOMs. In the following years the deployment rate should be 12-16 strings per season.

\section{Research topics}

Within the scope of this work only a selection of the topics being studied with AMANDA and IceCube can be presented.

The sky map as obtained from up-going tracks is given in figure 1. It is dominated by the roughly isotropically distributed muon tracks from neutrinos which originate from interactions of high energy cosmic rays in the Earth atmosphere. These so-called "atmospheric neutrinos" can be used to study systematics of the detector and to test models of cosmic ray interactions. In principle, they could also be used to study neutrino 
oscillation with a very long baseline, but the expected accuracy does not exceed that of the experiments dedicated to study neutrino oscillation.

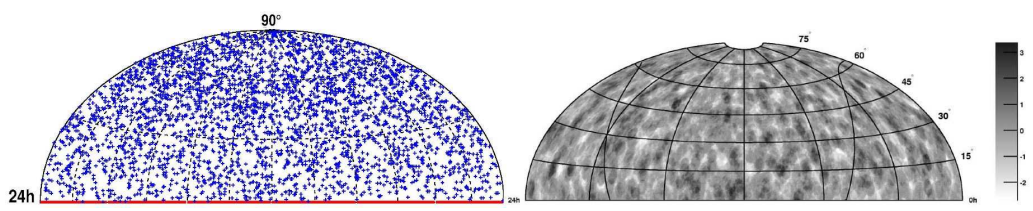

Figure 1. Left panel: directions of up-going muons with good reconstruction quality, from data taken in 2000-2003. Right panel: significance plot (gray-scale levels correspond to number of standard deviations above estimated background).

The sky map is analyzed for statistically significant excesses above this smooth atmospheric background. The total number of upward going tracks collected in 2000-2003 (807 days live-time) is 3329. The highest fluctuation was 3.4 sigma; the probability to get such an excess or greater in any angular bin in the sky map is $92 \%$. When restricting the search to the directions of a limited number of known celestial objects, the largest excess is 10 tracks pointing back to the Crab nebula, on an expected background of 5.4; the chance probability to find such an excess or greater for any of the 33 source candidates is $64 \%$. From these results upper limits can be derived for the neutrino fluxes from these sources ${ }^{7,8}$.

Another way to identify extraterrestrial neutrinos is by their energy, rather than their direction. The differential flux of atmospheric neutrinos measured in $\mathrm{AMANDA}^{3}$ is $\cos \theta \frac{d E}{d E} \propto E^{-3.54 \pm 0.11}$, whereas several models for extraterrestrial sources predict harder spectra ( $\propto E^{-2}$ or harder), which can be probed by restricting the search to higher energies at which we do expect very few atmospheric neutrinos. This is done by requiring a minimum number of PMTs with a signal. No excess over the expected background is observed yet.

If Nature is supersymmetric and R-parity is conserved, then the lightest supersymmetric particles (from now on "neutralinos") cannot decay, and we should expect a sizeable number of neutralinos to propagate freely through the Universe. They may lose energy through elastic scattering with ordinary matter and get gravitationally trapped inside a celestial body, for instance the Sun or the Earth. After losing more energy in subsequent elastic collisions they end up in the center of a celestial body, where the density of neutralinos increases until they have a finite chance of colliding with each other. They annihilate predominantly into $W^{+} W^{-}, b \bar{b}$ and $\tau \bar{\tau}$ 
which decay further into neutrinos and other particles. The rate at which AMANDA (and later IceCube) will detect such neutrinos from neutralino annihilation ${ }^{9}$ depends not only on the density and velocity distribution of neutralinos in the vicinity of the solar system, but also on their mass and their elastic scattering cross section with ordinary matter. These quantities vary between various versions of supersymmetric models. No excess rate of neutrinos has been observed with AMANDA from the direction of the Sun, nor from the center of the Earth. The mass dependent upper limits which we may derive from this ${ }^{10}$ on the neutralino density are $2-10$ times higher than the limits from direct searches for cold dark matter ${ }^{11}$. However, the indirect and direct methods are complementary since they apply to different parts of the neutralino velocity distribution.

\section{Conclusions}

AMANDA has successfully demonstrated the feasibility of neutrino detection with an array of optical modules in the Antarctic ice, and produced promising first results. The IceCube experiment is not only a larger scale detector, it also involves a major technological upgrade. IceCube, now partly constructed, is already the largest neutrino telescope in the world and will grow quickly in the next few years.

\section{References}

1. S. Yoshida et al., Astrophys. J. 479 (1997) 547.

2. K. Greisen, Phys. Rev. Lett. 16 (1966) 748; G.T.. Zatsepin and V.A. Kuzmin JETP Lett. 4 (1966) 178.

3. H. Geenen, Energy reconstruction and spectral unfolding of atmospheric leptons with the AMANDA detector, thesis Universität Wuppertal

4. M. Ackermann et al., Optical properties of deep glacial ice at the South Pole in J. Geophys. Res., in press, doi:10.1029/2005JD006687.

5. IceCube preliminary design report, see http://icecube.wisc.edu.

6. A. Achterberg et al., First Year Performance of the IceCube Neutrino Telescope, submitted to Astroparticle Physics.

7. M. Ackermann et al., Search for high energy neutrino point sources in the northern hemisphere with the AMANDA-II neutrino telescope, in Proc. of the 29th Int. Cosmic Ray Conf. (ICRC 2005), see also astro-ph/0509330.

8. M. Ackermann et al., Phys. Rev. D71 (2005) 077102.

9. W.H. Press and D.N. Spergel, Astrophys. J. 296 (1985) 679; J. Silk, K. Olive and M. Srednicki, Phys. Rev. Lett. 55 (1985) 257; T.K. Gaisser, G. Steigman and S. Tilav Phys. Rev. D34 (1986) 2206.

10. M. Ackermann et al., Astropart. Phys. 24 (2006) 459.

11. D.S. Akerib et al., astro-ph 0509269. 